\title{
A RADIAL MULTIPLIER AND A RELATED KAKEYA MAXIMAL FUNCTION
}

BY ANTONIO CORDOBA

Communicated by Alberto Calderón, November 11, 1974

In this paper we state some results for a maximal function and a Fourier multiplier that are connected with the Bochner-Riesz spherical summation of multiple Fourier series (see Fefferman [3], [5]). Our purpose will be to get sharp estimates for the norm of these operators in dimension two. Proofs will appear elsewhere [2].

Let $N \geqslant 1$ be a real number. By a rectangle of eccentricity $N$ we mean a rectangle $R$ such that

$$
\frac{\text { Length of the bigger side of } R}{\text { Length of the smaller side of } R}=N \text {. }
$$

We will define the direction of $R$ as the direction of its bigger side.

Given a locally integrable function $f$ we consider the maximal function

$$
M f(x)=\operatorname{Sup}_{x \in R} \frac{1}{|R|} \int_{R}|f(y)| d y,
$$

where the "Sup" is taken over rectangles of eccentricity $N$, but arbitrary direction.

THEOREM 1. The sublinear operator $M$ is bounded in $L^{2}\left(R^{2}\right)$ and there exists a constant $C$, independent of $N$, such that

$$
\|M f\|_{2} \leqslant C(\log 3 N)^{2}\|f\|_{2} .
$$

Suppose that $m_{0}$ is a smooth function on $R$ with support on $(-1,1)$ and let $m(r)=m_{0}\left(\delta^{-1}(r-1)\right)$, where $\delta>0$ is a small number.

Consider the Fourier multiplier defined by

$$
\widehat{T f}(\xi)=m(|\xi|) \hat{f}(\xi), \quad f \in C_{0}^{\infty}\left(R^{2}\right) .
$$

THEOREM 2. There exists a constant $C$, independent of $\delta$, such that $42 \mathrm{~A} 18$.

AMS (MOS) subject classifications (1970). Primary 42A68, 42A92; Secondary 


$$
\|T f\|_{4} \leqslant C\left\|\left.\log \delta\right|^{5 / 4}\right\| f \|_{4}, \quad \forall f \in C_{0}^{\infty}\left(R^{2}\right) .
$$

Our proofs of Theorems 1 and 2 are made in the spirit of Cotlar's lemma. In particular the support of the kernel of $T$ can be decomposed into a family of rectangles of eccentricity $\delta^{-1 / 2}$ and the convolution operators, obtained by restricting the kernel to these rectangles, are "almost orthogonal".

Theorem 2 can be applied to get Bochner-Riesz summability below the critical index, in dimension two.

Corollary 3 (Carleson-Sjolin-Fefferman-Hörmander). The

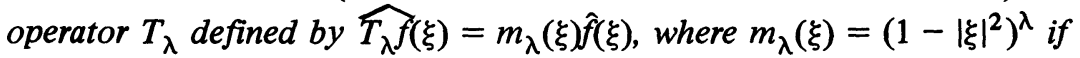
$|\xi| \leqslant 1$ and $m_{\lambda}(\xi)=0$ otherwise, is bounded in $L^{p}\left(R^{2}\right)$ if

$$
\frac{4}{3+2 \lambda}<p<\frac{4}{1-2 \lambda}, \frac{1}{2}>\lambda>0 \text {. }
$$

To see this we define a partition of unity on $(0,1)$ as follows: For every $n, h_{n}$ is a smooth function with support on $\left(1-2^{-n+1}, 1-2^{-n-1}\right)$ such that $\left|D^{p} h_{n}(r)\right| \leqslant A_{p} 2^{n p}$ (with $A_{p}$ independent of $n$ ) and $\Sigma h_{n}(r)=1$ on $(0,1)$. Then $m_{\lambda}(\xi)=\Sigma m_{\lambda}(\xi) h_{n}(|\xi|)$. If we apply Theorem 2 to the operator $T_{\lambda}^{n}$ defined by the multiplier $m_{\lambda}(\xi) h_{n}(|\xi|)$ we get that $\left\|T_{\lambda}^{n} f\right\|_{4} \leqslant$ $C 2^{-n \lambda} n^{5 / 4}\|f\|_{4}$. And then, Corollary 3 can be deduced from this estimate by standard arguments of interpolation, duality and adding a geometric series.

REMARK. Theorem 2 can be used to prove a sharper version of Corollary 3 i.e., suppose that $m$ is a smooth function on $(0,1)$ such that it behaves like

$$
\left(\log \frac{1}{1-|x|}\right)^{-t} \text { near }|x|=1 .
$$

Then $m$ is a multiplier for $L^{p}\left(R^{2}\right), 4 / 3 \leqslant p \leqslant 4$ provided that $t>9 / 4$.

I am deeply grateful to Professor Charles Fefferman for his many comments and criticisms, without which I could not have proved these results.

\section{REFERENCES}

1. L. Carleson and P. Sjolin, Oscillatory integrals and a multiplier problem for the disc, Studia Math. 44 (1972), 287-299.

2. A. Cordoba, The Kakeya maximal function and the spherical summation multipliers (to appear).

3. C. Fefferman, Inequalities for strongly singular convolution operators, Acta Math. 124 (1970), 9-36. MR 41 \#2468.

4. - The multiplier problem for the ball, Ann. of Math. (2) 94 (1971), 330-336. MR 45 \#5661. 
5. C. Fefferman, A note on spherical summation multipliers, Israel J. Math 15 (1973), 44-52. MR 47 \#160.

DEPARTMENT OF MATHEMATICS, UNIVERSITY OF CHICAGO, CHICAGO, ILLINOIS 60637

Current address: Department of Mathematics, Princeton University, Princeton, New Jersey 08540 